\title{
Unravel hepatic artery infusion chemotherapy in patients with resected colorectal liver metastases
}

\author{
Matteo Donadon ${ }^{1,2}$, Lorenza Rimassa $^{1,3}$, Guido Torzilli ${ }^{1,2}$ \\ ${ }^{1}$ Department of Biomedical Sciences, Humanitas University, Pieve Emanuele, Milan, Italy; ${ }^{2}$ Department of Hepatobiliary and General Surgery, \\ IRCCS Humanitas Research Hospital, Rozzano, Milan, Italy; ${ }^{3}$ Humanitas Cancer Center, IRCCS Humanitas Research Hospital, Rozzano, Milan, \\ Italy \\ Correspondence to: Matteo Donadon, MD, PhD. Department of Hepatobiliary and General Surgery, IRCCS Humanitas Research Hospital, Via \\ Manzoni 56, 20089 Rozzano, Milan, Italy. Email: matteo.donadon@hunimed.eu. \\ Comment on: Gholami S, Kemeny NE, Boucher TM, et al. Adjuvant Hepatic Artery Infusion Chemotherapy is Associated With Improved Survival \\ Regardless of KRAS Mutation Status in Patients With Resected Colorectal Liver Metastases: A Retrospective Analysis of 674 Patients. Ann Surg \\ 2020;272:352-6.
}

Submitted Dec 10, 2020. Accepted for publication Jan 11, 2021.

doi: $10.21037 / \mathrm{hbsn}-20-832$

View this article at: http://dx.doi.org/10.21037/hbsn-20-832

Over the past years, important progresses have been made in the treatment of patients with colorectal liver metastases (CLM). Now, 5-year overall survival rate may exceed $50 \%$ by using the combination of multimodality therapies among which modern systemic chemotherapy, with or without monoclonal antibodies, and hepatic resection are the cornerstones $(1,2)$. In such multimodality, hepatic artery infusion (HAI) has been proposed since many years with the rationale of increasing the concentration of chemotherapy agents in the liver (3). However, HAI still remains infrequently used, in particular in the adjuvant setting mainly because of the requirement of a multidisciplinary team to manage therapy, and the more widespread use of systemic chemotherapy-in particular following the perioperative "sandwich" schema (4).

On this line, we read with interest the retrospective study published by Gholami et al. (5), who recently reported on the utilization of adjuvant HAI in the setting of resected CLM. In particular, they reported how adjuvant HAI was independently associated with improved recurrencefree and overall survival regardless of KRAS mutational status. Consistently with their good results, they also suggested that the use of HAI might be used to mitigate the anticipated worse outcome in KRAS mutated in comparison with KRAS wild-type patients.

The long-standing work on HAI from the multidisciplinary group of Memorial Sloan Kettering
Cancer Center of New York City is commendable. Since 1992 (6), these authors have published many studies in support on the use of HAI in CLM patients. However, to stay on the most recent work we would like to report how these results should be read with caution because of the consistent risk of selection bias.

The authors did not report any information about NRAS, BRAF, microsatellite status, and other biomarkers that might have had an impact on the results of the analysis. In particular, the correlation between BRAF status, RAS status and outcome is well-known, with BRAF mutated tumors being associated to the worst prognosis, RAS wildtype to the best, and RAS mutant to an intermediate one, although many other factors should be considered in defining patients' prognosis. In addition, on a total of 2,690 patients with CLM who underwent surgery only $25 \%$ had known mutational KRAS status and no details are available for these patients compared to the patients with unknown RAS status. Besides, the heterogeneity in the methods used to obtain KRAS mutational status should be mentioned as a further potential confounding factor.

Importantly, $46 \%$ of the patients did not receive HAI and no information about the selection criteria were provided. HAI was generally not offered to patients with progressive disease, however, a small minority of selected cases, as acknowledged by the authors, received HAI therapy notwithstanding disease progression, and it would be of 
Table 1 Trials of adjuvant hepatic arterial infusion

\begin{tabular}{|c|c|c|c|c|c|}
\hline Author, year & Patients No. & Regimen & OS & RFS & Notes \\
\hline Kemeny MM et al. 2002 & 109 & HAI FUDR + systemic 5FU vs. no therapy & $\mathrm{n} / \mathrm{a}$ & $\begin{array}{c}\text { 4-year: } 46 \% \\
\text { vs. } 25 \%\end{array}$ & - \\
\hline
\end{tabular}

OS, overall survival; RFS, recurrence-free survival; HAI, hepatic arterial infusion; 5FU, 5-fluorouracil; LV, leucovorin; n/a, not available;

FUDR, floxuridine; IL-2, interleukin 2.

interest to have some details about these patients. Did they have a biologically more favorable profile and therefore a better prognosis compared to patients with progressive disease who did not receive HAI?

Further important aspects to be mentioned are represented by the inclusion in the study of patients with synchronous and metachronous CLM, with initially unresectable and resectable disease, and the lack of details about the primary tumor sidedness, all being related to a different prognosis and representing relevant confounding factors. Indeed, available evidence shows that the abovementioned subgroups of patients have different prognoses and benefit from different therapeutic approaches. Systemic doublet chemotherapy (FOLFOX/CAPOX) in combination with resection is recommended for resectable CLM, while doublet or triplet (FOLFOXIRI) chemotherapy with or without the addition of a monoclonal antibody is, in general, the choice for initially unresectable CLM.

Further issues should be noted: (I) the study period (1993-2012) includes patients treated many years ago, when the modern systemic chemotherapy was not really modern; the modern FOLFOX/CAPOX and FOLFIRI regimes with or without anti-vascular endothelial growth factor or anti-epidermal growth factor receptor (EGFR) agents were adopted since the early 2000s in North America and in Europe; therefore, the patients included in this study received different regimens over the study period and no details of the type of administered systemic therapy are provided; (II) the use of neoadjuvant plus adjuvant or adjuvant-only systemic chemotherapy has not been detailed either as indication criteria, as number of treated patients per group, as type of regimens, as number of courses and as radiological response to such treatments; (III) similarly, the use of HAI as neoadjuvant plus adjuvant or adjuvant-only has not been detailed as indication criteria, number and duration of courses; (IV) the rate of extrahepatic diseases in no-HAI was double than the rate in HAI group (14\% vs. 7\%); consistently, the rate of extrahepatic recurrence after hepatectomy was increased in no-HAI group, even if it did not reach the statistical significance; (V) the current standard practice is to treat RAS wild-type patients with left-sided cancer by using anti-EGFR agents; thus, it would be of interest to assess the effect of adjuvant HAI in this subgroup of patients treated also with systemic chemotherapy combined with an anti-EGFR agent over the course of their disease.

Although the rationale for adjuvant HAI is intuitive, since approximately $70 \%$ of patients develop intrahepatic recurrence during the follow-up (2), several randomized control trials (RCTs) demonstrated mixed results (Table 1): three studies showed increase in overall and/or recurrencefree survival with the addition of HAI to surgery with or without systemic chemotherapy, while one study was early interrupted because of no difference (7-10). Of note, the two studies that showed an advantage in recurrencefree survival were from the same group $(8,9)$. Besides, a Cochrane review summarizing the data from approximately 600 patients found no significant differences in overall survival between no-HAI $v s$. HAI (11). Even if differences in types of administered agents and in concurrent systemic therapy among centers limit generalizability, to date HAI should be considered only as an option in selected patients treated in expert centers. Indeed, the current guidelines from the European Society for Medical Oncology do not recommend HAI (12), while the guidelines from the National Comprehensive Cancer Network suggest the use of HAI as an alternative to systemic therapy in patients with limited liver-only disease (13). 
Currently two phase III RCTs of adjuvant HAI are underway. The first is the PUMP trial from the Netherlands that aims to investigate recurrence-free survival in patients at low risk of recurrence being treated by resection only $v s$. resection plus HAI (14). The second is the PACHA-01 trial from France that conversely aims to investigate recurrencefree survival in patients at high risk of recurrence being treated by FOLFOX $v s$. HAI with oxaliplatin plus systemic 5 -flourouracil and leucovorin (15). Both these studies will give us evidence on potential benefits of adjuvant HAI but the one conducted in high-risk patients, which includes patients with at least 4 CLM and treated also with R1hepatectomy and/or thermo-ablation, is particularly awaited because includes those patients routinely treated in highvolume centers.

As clinicians we appreciate that a high level of evidence cannot always be achieved with RCTs and in specific settings, as in case of liver resection for CLMs, large case series and observational studies might be useful and should be considered. However, in such retrospective study the weight of different patients' characteristics and of therapeutic regimens, as well as risk stratification, should be given to support important conclusions and make the evidence of survival benefits from adjuvant HAI stronger.

\section{Acknowledgments}

Funding: None.

\section{Footnote}

Provenance and Peer Review: This article was commissioned by the editorial office of Hepatobiliary Surgery and Nutrition. The article did not undergo external peer review.

Conflicts of Interest: All authors have completed the ICMJE uniform disclosure form (available at https://hbsn.amegroups. com/article/view/10.21037/hbsn-20-832/coif). Dr. LR reports personal fees from Amgen, ArQule, AstraZeneca, Basilea, Bayer, BMS, Celgene, Eisai, Exelixis, Genenta, Hengrui, Incyte, Ipsen, IQVIA, Lilly, MSD, Nerviano Medical Sciences, Roche, Sanofi, personal fees from AbbVie, Amgen, Eisai, Gilead, Incyte, Ipsen, Lilly, Merck Serono, Roche, Sanofi, non-financial support from Ipsen, other from Agios, ARMO BioSciences, AstraZeneca, BeiGene, Eisai, Exelixis, Fibrogen, Incyte, Ipsen, Lilly, MSD, Nerviano Medical Sciences, Roche, Zymeworks, outside the submitted work. The other authors have no conflicts of interest to declare.
Ethical Statement: The authors are accountable for all aspects of the work in ensuring that questions related to the accuracy or integrity of any part of the work are appropriately investigated and resolved.

Open Access Statement: This is an Open Access article distributed in accordance with the Creative Commons Attribution-NonCommercial-NoDerivs 4.0 International License (CC BY-NC-ND 4.0), which permits the noncommercial replication and distribution of the article with the strict proviso that no changes or edits are made and the original work is properly cited (including links to both the formal publication through the relevant DOI and the license). See: https://creativecommons.org/licenses/by-nc-nd/4.0/.

\section{References}

1. Choti MA, Sitzmann JV, Tiburi MF, et al. Trends in long-term survival following liver resection for hepatic colorectal metastases. Ann Surg 2002;235:759-66.

2. Cucchetti A, Ferrero A, Cescon M, et al. Cure model survival analysis after hepatic resection for colorectal liver metastases. Ann Surg Oncol 2015;22:1908-14.

3. Dizon DS, Schwartz J, Kemeny N. Regional chemotherapy: a focus on hepatic artery infusion for colorectal cancer liver metastases. Surg Oncol Clin N Am 2008;17:759-71, viii.

4. Karanicolas PJ, Metrakos P, Chan K, et al. Hepatic arterial infusion pump chemotherapy in the management of colorectal liver metastases: expert consensus statement. Curr Oncol 2014;21:e129-36.

5. Gholami S, Kemeny NE, Boucher TM, et al. Adjuvant Hepatic Artery Infusion Chemotherapy is Associated With Improved Survival Regardless of KRAS Mutation Status in Patients With Resected Colorectal Liver Metastases: A Retrospective Analysis of 674 Patients. Ann Surg 2020;272:352-6.

6. Kemeny NE. Is hepatic infusion of chemotherapy effective treatment for liver metastases? Yes!. Important Adv Oncol 1992;207-27.

7. Lorenz M, Müller HH, Schramm H, et al. Randomized trial of surgery versus surgery followed by adjuvant hepatic arterial infusion with 5 -fluorouracil and folinic acid for liver metastases of colorectal cancer. German Cooperative on Liver Metastases (Arbeitsgruppe Lebermetastasen). Ann Surg 1998;228:756-62.

8. Kemeny MM, Adak S, Gray B, et al. Combined-modality treatment for resectable metastatic colorectal carcinoma 
to the liver: surgical resection of hepatic metastases in combination with continuous infusion of chemotherapy-an intergroup study. J Clin Oncol 2002;20:1499-505.

9. Kemeny N, Huang Y, Cohen AM, et al. Hepatic arterial infusion of chemotherapy after resection of hepatic metastases from colorectal cancer. $\mathrm{N}$ Engl J Med 1999;341:2039-48.

10. Lygidakis NJ, Sgourakis G, Vlachos L, et al. Metastatic liver disease of colorectal origin: the value of locoregional immunochemotherapy combined with systemic chemotherapy following liver resection. Results of a prospective randomized study. Hepatogastroenterology 2001;48:1685-91.

11. Nelson R, Freels S. Hepatic artery adjuvant chemotherapy for patients having resection or ablation of colorectal cancer metastatic to the liver. Cochrane Database Syst Rev 2006;(4):CD003770.

12. Van Cutsem E, Cervantes A, Nordlinger B, et al.

Cite this article as: Donadon M, Rimassa L, Torzilli G. Unravel hepatic artery infusion chemotherapy in patients with resected colorectal liver metastases. HepatoBiliary Surg Nutr 2021;10(2):257-260. doi: 10.21037/hbsn-20-832
Metastatic colorectal cancer: ESMO Clinical Practice Guidelines for diagnosis, treatment and follow-up. Ann Oncol 2014;25 Suppl 3:iii1-iii9. Erratum in: Ann Oncol. 2015 Sep;26 Suppl 5:v174-7. doi: 10.1093/annonc/ mdv204. Epub 2015 Jul 21.

13. National Comprehensive Cancer Network. Available online: https://www.nccn.org/professionals/physician_gls/ default.aspx

14. Buisman FE, Homs MYV, Grünhagen DJ, et al. Adjuvant hepatic arterial infusion pump chemotherapy and resection versus resection alone in patients with low-risk resectable colorectal liver metastases - the multicenter randomized controlled PUMP trial. BMC Cancer 2019;19:327.

15. Goéré D, Pignon JP, Gelli M, et al. Postoperative hepatic arterial chemotherapy in high-risk patients as adjuvant treatment after resection of colorectal liver metastases - a randomized phase II/III trial - PACHA-01 (NCT02494973). BMC Cancer 2018;18:787. 